\title{
Analysis of stability levee during different flooding wave stages
}

\author{
Maciej Dwornik ${ }^{1}$, Anna Franczyk ${ }^{1}$, Krzysztof Krawiec ${ }^{2}$ \\ ${ }^{1}$ AGH University of Science and Technology, Faculty of Geology, Geophysics and Environmental Protection, \\ Department of Geoinformatics and Applied Computer Science; al. A. Mickiewicza 30, 30-059 Krakow, Poland; \\ e-mail:dwornik@geol.agh.edu.pl,franczyk@geol.agh.edu.pl \\ ${ }^{2}$ The Mineral and Energy Economy Research Institute of the Polish Academy of Sciences; ul. Wybickiego 7, 31-261 Krakow, \\ Poland;e-mail:k.krawiec@meeri.eu
}

(C) 2016 Authors. This is an open access publication, which can be used, distributed and reproduced in any medium according to the Creative Commons CC-BY 4.0 License requiring that the original work has been properly cited.

The stability of levee depends mainly on mechanical and hydromechanical properties of material used for its construction. The structural capacity of a system beyond the expected loads or actual loads is calculated to a variety of different underground structures such as slopes, retaining walls or tunnels. The stability of structure is often expressed by Factor of Stability (FoS) obtained by numerical modeling. In case of the stability analysis of the levee, the flooding process is limited to few scenarios of flooding waves. The first approach of determination of the most significant stages of flooding wave shape can be found in Dwornik et al. (2015).

In this paper, the stability of earthen levee for different stages and flooding waves was calculated. Stability of the levee was described using Factor of Safety implemented in Itasca Flac 2D 7.0 software using the strength reduction procedure (Itasca 2011). It is applied by a strength reduction method, which reduces the shear strength of the material to bring the slope to a state of limiting equilibrium. The value of FoS bigger than 1.0 indicate the stable construction, whereas the value less than 1.0 should be interpreted as a possible unstable model (Itasca 2011).

Numerical modeling presented in this paper was performed for 17 different schemes of flooding wave. All the schemas were slight modification of the flooding wave considered during the ISMOP project (www.ismop.pl, Mościcki et al. 2014). The analysis was conducted to the flooding wave described by the four stages: water level increasing, durability of the height water level, the pace of water level decreasing and durability of the stage between cycles of successive flooding waves. The increasing of the water level assumed in the numerical modeling varied from $0.1 \mathrm{~m}$ to $3.5 \mathrm{~m}$ in height. The high water level, assumed as a $3.5 \mathrm{~m}$, was different for the individual scenario. The decreasing water level varied from $3.5 \mathrm{~m}$ to $0.1 \mathrm{~m}$ preceding the low water level assumed $0.1 \mathrm{~m}$ in height.

Calculations show that levee is stable for all tested construction stages. The lowest FoS value was obtained after decreasing water level in the reservoir between levees. The slowest pace of decreasing water level was assumed, the higher value of FoS was obtain, which indicated better levee stability.

The study was partly financed from the statutory research project No. 11.11.140.613 of the Department of Geoinformatics and Applied Computer Science, AGH University of Science and Technology and by the National Centre for Research and Development (NCBiR) in Poland, project PBS1/B9/18/2013 (no 180535).

\section{REFERENCES}

Dwornik M., Krawiec K., Pięta A. \& Leśniak A., 2015. Numerical and experimental stability analysis of earthen levees. [in:] IAMG 2015: the 17th annual conference of the International Association for Mathematical Geosciences: 
Freiberg, Germany, September 5-13, 2015: short abstracts, IAMG, 857-864.

Itasca Consulting Group, 2011. FLAC Fast Lagrangian Analysis of Continua - User's Manual. Minneapolis, USA.
Mościcki J.W., Bania G., Cwiklik M. \& Borecka A., 2014. DC resistivity studies of shallow geology in the vicinity of Vistula river flood bank in Czernichów Village (near Kraków in Poland). Studia Geotechnica et Mechanica, $36,1,63-70$. 